\title{
Neuroprotective effects of lycopene pretreatment on transient global cerebral ischemia-reperfusion in rats: The role of the Nrf2/HO-1 signaling pathway
}

\author{
XIAOFENG LEI ${ }^{1}$, LIJIAN LEI ${ }^{2}$, ZHELIN ZHANG $^{1}$ and YAN CHENG ${ }^{1}$ \\ ${ }^{1}$ Department of Neurology, Tianjin Fourth Center Hospital, Tianjin 300140; \\ ${ }^{2}$ Deparment of Teaching and Research Section of Epidemiology, Shanxi Medical University, \\ Taiyuan, Shanxi 030001, P.R. China
}

Received March 22, 2015; Accepted October 9, 2015

DOI: $10.3892 / \mathrm{mmr} .2015 .4534$

\begin{abstract}
The present study aimed to investigate the neuroprotective effect of lycopene in a mouse model of bilateral common carotid artery occlusion (BCCAO) and the role of the Nrf2/HO-1 signaling pathway. A total of 60 male C57BL/6 mice, aged 12 weeks and weighing 20-24 g, were used in the present study. The mice were randomly assigned to three groups: Control, BCCAO and BCCAO + lycopene. The neurological score was assessed 24,48 or $72 \mathrm{~h}$ following BCCAO. Hematoxylin and eosin staining, and terminal deoxynucleotidyl transferase dUTP nick end labeling (TUNEL) were performed to detect neuronal death and survival. The production of glutathione (GSH) and reactive oxygen species were detected to investigate the oxidative stress. The expression levels of nuclear factor erythroid 2-related factor (Nrf2) and Heme oxygenase-1 (HO-1) were determined by western blotting. Lycopene significantly improved the neurological score in the BCCAO mice. It attenuated neuronal apoptosis, as indicated by TUNEL staining, and attenuated the oxidative stress induced by global ischemia. Lycopene increased the expression levels of Nrf2 and HO-1, indicating that the Nrf2/HO-1 signaling pathway may be involved in the neuroprotective effect of lycopene. The present study revealed that lycopene protects the brain from global ischemic injury, which is associated with its antiapoptotic effect and the activation of the Nrf2/HO-1 signaling pathway.
\end{abstract}

\section{Introduction}

Ischemic stroke is currently a leading cause of cerebrovascular disease worldwide, and exhibits a high morbidity and

Correspondence to: Dr. Xiaofeng Lei, Department of Neurology, Tianjin Fourth Center Hospital, 1 Zhongshan Road, Tianjin 300140, P.R. China

E-mail: leixf2014@163.com

Key words: lycopene, apoptosis, global cerebral ischemia, $\mathrm{Nrf} 2 / \mathrm{HO}-1$ mortality among patients (1). Ischemic stroke is induced by a transient or permanent occlusion in the cerebral vessel, resulting in neuronal death and associated behavioral deficits, including sensorimotor dysfunction, spatial orientation disorder, and learning and memory impairment (2-4). In addition, the mechanisms underlying stroke include oxidative stress, blood-brain barrier dysfunction, neuronal apoptosis and inflammation $(5,6)$. Although tissue-type plasminogen activator is used clinically and remains the only FDA-approved treatment for ischemic stroke, it is not so effective for all patients and only a small number of patients recover as a result of the reperfusion injury and a narrow $3 \mathrm{~h}$ time-window for safe administration (7). Therefore, other effective therapeutic agents are required to assist the patients with their diseases.

Lycopene, a member of the carotenoid family, is found predominantly in tomatoes and other red colored fruits (8). It has been previously reported that lycopene has several biological functions in various diseases. Lycopene protects the cell from lipid peroxidation and oxidative DNA damage as a highly efficient antioxidant $(8,9)$. In addition, lycopene exhibits other properties, including antiapoptosis (10), anti-inflammation $(11,12)$, antiamyloid (13), anti-ischemia (14), and antitumor properties (15). Since lycopene has a high liposolubility, it can cross the blood-brain barrier (16). It has also been demonstrated that lycopene is beneficial for certain neurological disorders, including Alzheimer's disease $(17,18)$. Therefore, lycopene is potentially beneficial in other brain diseases and the present study set out to investigate this.

Oxidative stress is important in ischemic stroke, characterized by a dramatic increase in reactive oxygen species (19). In normal cells, the antioxidant system protects cells from various oxidative stresses. Antioxidant/electrophile response element (ARE)-regulated phase II detoxifying enzymes and antioxidants are one of the predominant antioxidant pathways involved in attenuating increased oxidative stress and maintaining the redox status in several tissues and organs (20). Heme oxygenase-1 (HO-1) is an ARE-regulated enzyme and antioxidant, which is regulated by the redox-sensitive transcription factor, nuclear factor erythroid 2-related factor (Nrf2) (21). The function of HO-1 is to catalyze heme to biliverdin, carbon monoxide and iron. It has been previously reported that Nrf-2 
activation protects the neurons from ischemia (22). Under physiological conditions, Nrf2 is located in the cytosol and binds to Kelch-like ECH-associated protein 1 (Keap1). In response to oxidative stress, Nrf2 dislocates from Keap1 and translocates to the nucleus $(23,24)$, where it forms a heterodimer with its obligatory partner, Maf, and binds to the ARE sequence to activate the transcription of numerous antioxidative and electrophile detoxification genes, including $\mathrm{HO}-1, \mathrm{NAD}(\mathrm{P}) \mathrm{H}$ :quinine oxidoreductase 1 and glutamate-cysteine ligase (25).

The present study aimed to investigate whether lycopene exerts a neuroprotective effect on the ischemic brain in a bilateral common carotid artery occlusion (BCCAO) model. If so, the present study aimed to determine whether it regulates Nrf2/HO-1 signaling in this ischemic model.

\section{Materials and methods}

Animals. A total of 60 C57BL/6 mice, aged 12 weeks and weighing 20-24 g, were used in the experiments and were provided by the Experimental Animal Center of the Tianjin Medical University (Tianjin, China). The mice were maintained in cages under a controlled-light environment $(12 \mathrm{~h}$ light/dark cycles) and were allowed free access to a rodent diet and tap water. The present study was approved by the Ethics Committee of Tianjin Medical University. All animals used in this study were cared for in accordance with the Guidance for the Care and Use of Laboratory Animals published by the United States National Institute of Health.

Establishment of global cerebral ischemia. BCCAO was used as a model of global cerebral ischemia, as previously reported (26). Surgical operation was performed by an individual in a blinded manner. The mice were anesthetized with 3\% isoflurane (Baxter, Deerfield, IL, USA). Following induction, the concentration of isoflurane was maintained at $1.5 \%$. Isoflurane was administered via a face mask, which was constructed to fit over the animals' frontal area. A midline incision was made to the region between the neck and sternum to expose the trachea. The right and left common carotid arteries were located lateral to the sternocleidomastoid and were carefully separated. Cerebral ischemia was induced by clamping each of the arteries with two miniature artery clips. Following $20 \mathrm{~min}$ of cerebral ischemia, the clips were removed from each artery to allow for the reperfusion of blood through the carotid arteries. Sham-operated mice underwent the identical surgical procedure without artery occlusion. During the surgical procedure, the pericranial temperature was monitored using a temperature probe and maintained at $37.0-37.5^{\circ} \mathrm{C}$ using a heating pad. Following surgery, the animals were placed in a warm environment $\left(30-33^{\circ} \mathrm{C}\right)$ to avoid biased results due to hypothermia.

Drug administration. For the BCCAO + lycopene group, lycopene was intraperitoneally administered at a dose of $20 \mathrm{mg} / \mathrm{kg}$ for seven consecutive days prior to surgery. The mice in the sham group and BCCAO group were injected solely with an equal concentration of dimethyl sulfoxide (DMSO). Lycopene was dissolved in $2 \%$ DMSO.

Neurological tests. The treated mice were allowed to recover for $24 \mathrm{~h}$ prior to subsequent tests. The mice were subjected to a modified neurological examination designed to detect motor deficits. Briefly, the mice were placed on a $10-20 \mathrm{~cm}$ screen (grid size $0.2 \times 0.2 \mathrm{~cm}$ ), which can be rotated from $0^{\circ}$ (horizontal) to $90^{\circ}$ (vertical). The mice were placed on this screen, which was in a horizontal position, and the screen was then rotated into the vertical plane. The duration for which each mouse was able to hold on to the vertical screen was recorded up to a maximum of $15 \mathrm{sec}$ (corresponding to a maximum of three points). Next, the mouse was placed at the center of a horizontal wooden rod (diameter, $1.5 \mathrm{~cm}$ ), and the duration that the mouse was able to remain balanced on the rod was recorded up to a maximum of $30 \mathrm{sec}$ (corresponding to a maximum of three points). Finally, a prehensile traction test was performed. The duration that the mouse was able to cling to a horizontal rope was recorded up to a maximum of $5 \mathrm{sec}$ (corresponding to a maximum of three points). From these tests, a total motor score (TMS; nine possible points) was calculated. The neurological assessments were performed at 24,48 or $72 \mathrm{~h}$ post-reperfusion by an observer in a blinded manner. The TMS has been shown previously to be an accurate method for evaluating global cerebral ischemic injury in mice (27).

Hematoxylin and eosin (HE) staining. Neuronal damage was assessed using HE staining (Beyotime Institute of Biotechnology, Shanghai, China). On day 3 following the induction of ischemia, the animals were anesthetized with sodium pentobarbital $(50 \mathrm{mg} / \mathrm{kg}$ intraperitoneally; Sigma-Aldrich, St. Louis, MO, USA) and transcardially perfused with $4 \%$ phosphate-buffered paraformaldehyde, following a flush with $0.1 \mathrm{M}$ phosphate-buffered saline (PBS). The brains were removed, post-fixed at $4^{\circ} \mathrm{C}$ in $4 \%$ paraformaldehyde overnight and then sectioned on a freezing microtome. The brains were sectioned backward from the optic chiasm into six consecutive sections $(12 \mu \mathrm{m})$, which included the dorsal hippocampus, and were stained with HE. The pyramidal neurons of the CA1 region were examined.

Terminal deoxynucleotidyl transferase dUTP nick end labeling (TUNEL). The tissue sections were placed on slides and incubated with TUNEL reaction mixture (Roche Diagnostics GmbH, Mannheim, Germany), including enzyme solution (terminal deoxynucleotidyl transferase) and tetramethylrhod-amine-labeled TUNEL-positive nucleotides, in a dark humidified chamber for $1 \mathrm{~h}$ at $37^{\circ} \mathrm{C}$, followed by a final wash for $3 \times 10$ min with PBS and then covered with water-based mounting medium (National Diagnotics, Atlanta, GA, USA). The captured images were viewed and analyzed using laser scanning confocal microscopy (FV1000; Olympus, Tokyo, Japan).

Western blot analysis. Proteins were extracted following brain tissue homogenization in radioimmunoprecipitation acid buffer (EMD Millipore, Billerica, MA, USA). The total protein content was determined using a bicinchoninic acid protein assay. The protein samples $(50 \mu \mathrm{g})$ were separated by electrophoresis on SDS-PAGE gels (Beijing Solarbio Science $\&$ Technology Co., Ltd., Beijing, China) and were transferred onto polyvinylidene difluoride membranes (EMD Millipore). The membranes were blocked with $5 \%$ non-fat milk at room 
A

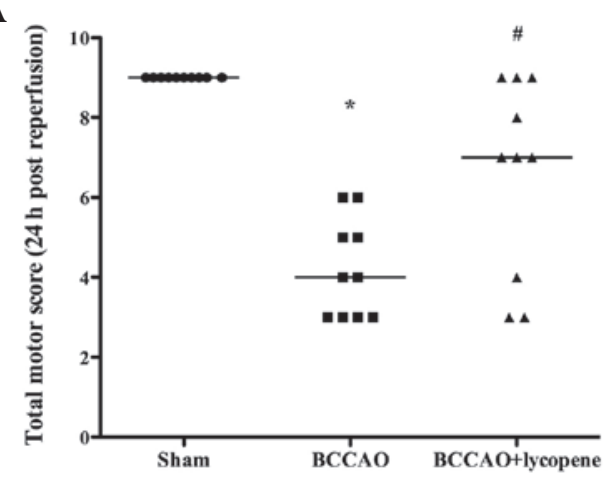

B

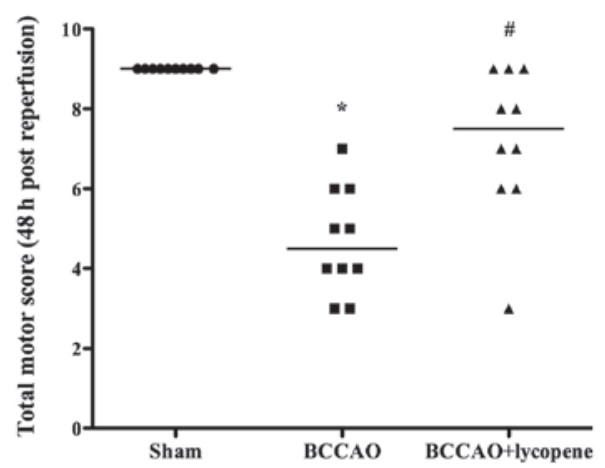

C

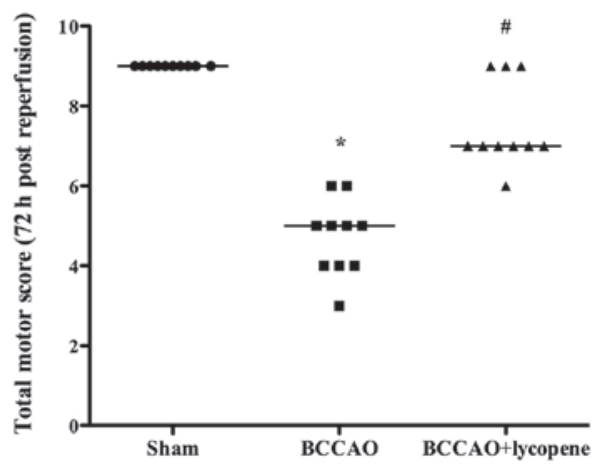

Figure 1. Neurological scores of each animal recorded at (A) 24, (B) 48 or (C) $72 \mathrm{~h}$ following reperfusion. The total motor scores in the BCCAO + lycopene group were significantly improved compared with those in $\mathrm{BCCAO}$ groups at 24,48 or $72 \mathrm{~h}$ following reperfusion $\left(\mathrm{n}=10 ;{ }^{*} \mathrm{P}<0.05 \mathrm{vs}\right.$. Sham group; ${ }^{\prime \prime} \mathrm{P}<0.05$ vs. $\mathrm{BCCAO}$ group). BCCAO, bilateral common carotid artery occlusion.

temperature for $2 \mathrm{~h}$ and were incubated overnight with the appropriate primary antibodies. The antibodies used were rabbit polyclonal anti-Nrf2 (1:200; cat. no. sc-13032; Santa Cruz Biotechnology, Inc., Santa Cruz, CA, USA), rabbit polyclonal anti-HO-1 (1:200; cat. no. sc-10789; Santa Cruz Biotechnology, Inc.), rabbit polyclonal anti-Histone1 (1:1,000; cat. no. ab4270; Abcam, Cambridge, MA, USA), and mouse monoclonal anti- $\beta$-actin $(1: 5,000$; cat. no. sc-47778; Santa Cruz Biotechnology, Inc.). Following extensive rinsing with Tris-buffered saline, containing $0.1 \%$ Triton X-100 buffer, the membranes were incubated with mouse anti-rabbit (cat. no. sc-2357) and goat anti-mouse (cat. no. sc-2005) horseradish peroxidase-conjugated secondary antibodies (1:2,000; Santa Cruz Biotechnology, Inc.) for $1 \mathrm{~h}$ at room temperature. The membranes were developed and a bar graph was produced to depict the ratios of semi-quantitative results obtained by scanning reactive bands and quantifying the optical density using Image Lab version 4.0 software (Bio-Rad Laboratories, Hercules, CA, USA).

Statistical analysis. All statistical analyses were performed using SPSS 11.0 for Windows software (SPSS, Inc., Chicago, IL, USA). All values, with the exception of TMS, are presented as the mean \pm standard error of the mean, and were analyzed using a one-way analysis of variance. Between-groups, differences were detected based on post-hoc Student-Newman-Keuls tests. The TMS are expressed as the medians and were analyzed using the Kruskal-Wallis test. $\mathrm{P}<0.05$ was considered to indicate a statistically significant difference.

\section{Results}

Neurological score. As shown in Fig. 1, the neurological score in the BCCAO group markedly decreased compared with that in the sham group $(\mathrm{P}<0.05)$ at 24,48 and $72 \mathrm{~h}$ following the induction of ischemia. Lycopene treatment ameliorated the injury and the neurological score was increased compared with that in the BCCAO group $(\mathrm{P}<0.05)$.

$H E$ staining. A total of 3 days following reperfusion, the number of viable neurons in the CA1 region was markedly decreased in the BCCAO group. Lycopene treatment significantly reduced the neuronal degeneration in the CA1 region compared with that in the BCCAO group (Fig. 2).

TUNEL. As shown in Fig. 3, ischemia induced a marked neuronal apoptotic response compared with the sham group. The survival of the neurons was markedly increased when lycopene was administered, suggesting that lycopene treatment attenuated the apoptosis of neurons, as indicated by the decrease in the number of TUNEL-positive neurons in the CA1 region.

Oxidative stress. Global cerebral ischemia induced a dramatic decrease in the production of GSH and a significant increase in the production of reactive oxygen species (ROS). When lycopene was administrated, the production of GSH was increased $(\mathrm{P}<0.05)$ and the production of ROS was decreased $(\mathrm{P}<0.05)$, indicating that lycopene protects the ischemic brain from oxidative stress (Fig. 4). 
A

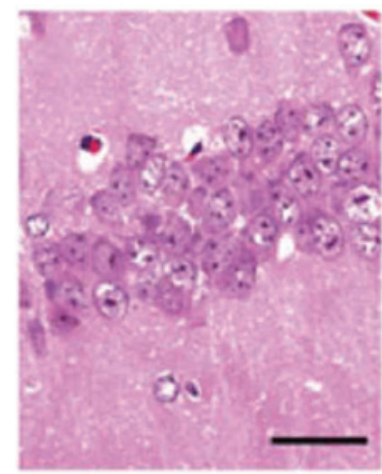

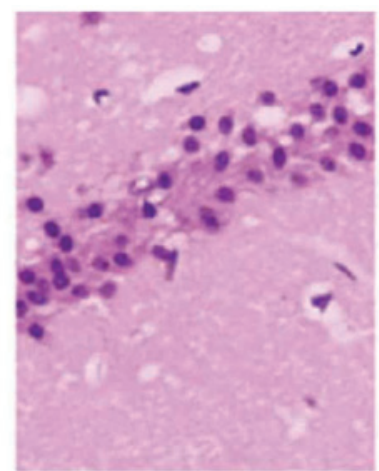

C

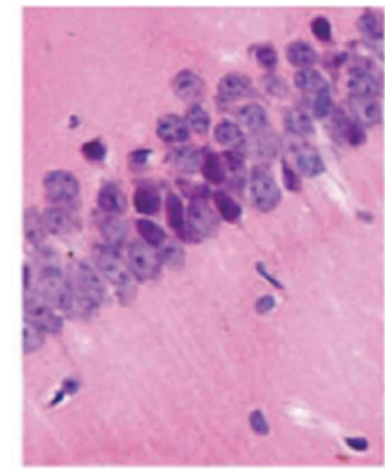

D

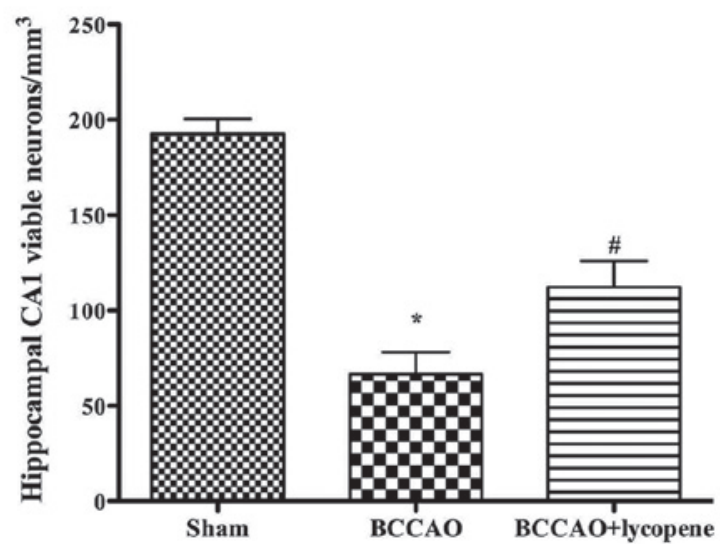

Figure 2. Lycopene treatment protects hippocampal CA1 neurons against ischemic injury. (A-C) Representative microphotographs of hematoxylin-eosin-stained neurons in hippocampal CA1 regions at $72 \mathrm{~h}$ following reperfusion in mice. The (A) Sham, (B) BCCAO and (C) BCCAO + lycopene groups following $72 \mathrm{~h}$ experiments are shown. (D) Viable CA1 neurons were quantified and analyzed at $72 \mathrm{~h}$ following reperfusion. Viable neurons were significantly decreased in the $\mathrm{CA} 1$ region in the $\mathrm{BCCAO}$ group compared with those in the sham group, whereas viable neurons were significantly increased the $\mathrm{BCCAO}+\mathrm{lycopene}$ group compared with those in the BCCAO group at $72 \mathrm{~h}$ following reperfusion $\left(\mathrm{n}=10 ;{ }^{*} \mathrm{P}<0.05\right.$ vs. Sham group; ${ }^{*} \mathrm{P}<0.05$ vs. BCCAO group). BCCAO, bilateral common carotid artery occlusion.

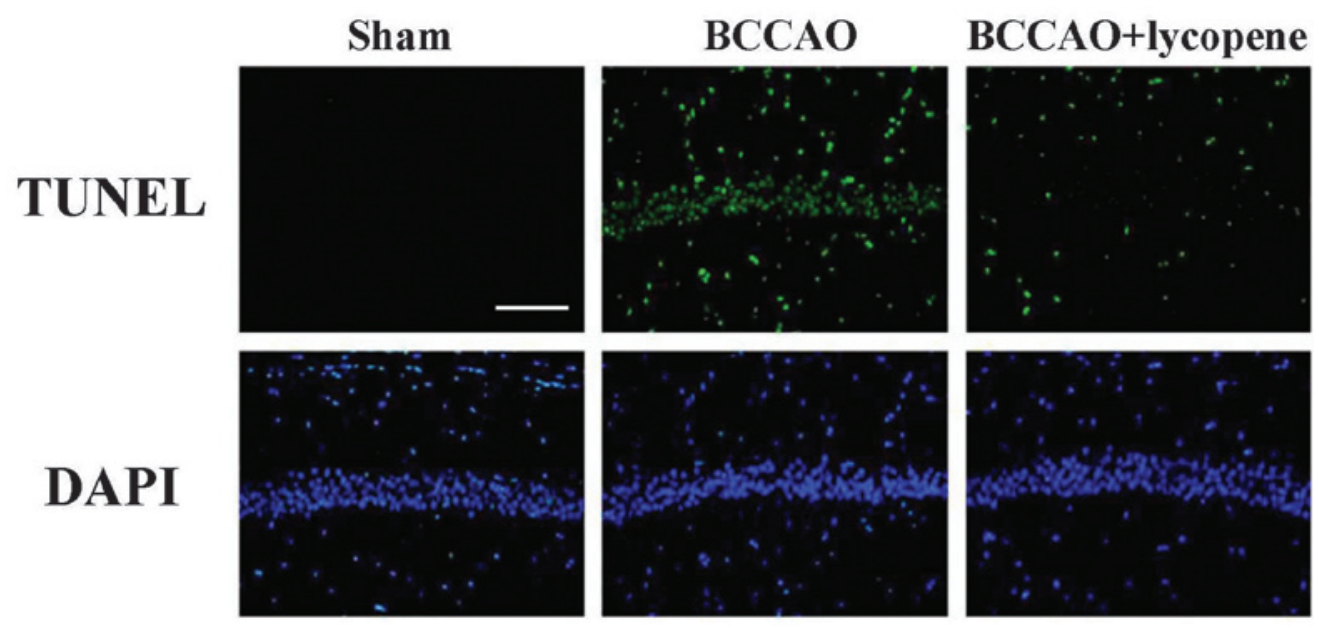

Figure 3. Resveratrol preconditioning significantly attenuates neuronal apoptosis in the hippocampal CA1 region. Representative images of TUNEL staining are shown. The results suggested that neuronal apoptosis was increased dramatically in the BCCAO group compared with the sham group, whereas lycopene treatment significantly attenuated neuronal apoptosis compared with the BCCAO group (Scale bar=50 $\mu \mathrm{m} ; \mathrm{n}=10$ ). BCCAO, bilateral common carotid artery occlusion; DAPI, 4',6-diamidino-2-phenylindole; TUNEL, terminal deoxynucleotidyl-transferase-mediated dUTP nick end labeling.

Effect of lycopene on the expression of $\mathrm{Nrf} 2$ and $\mathrm{HO}-1$. As shown in Figs. 5 and 6, the expression levels of nuclear and total Nrf2 and HO-1 in the hippocampus were detected by western blotting. The nuclear and total Nrf2 were markedly upregulated in the lycopene treatment group. In addition, lycopene significantly upregulated the expression of HO-1.

\section{Discussion}

The results of the present study demonstrated that lycopene preconditioning has a neuroprotective effect in cerebral ischemia-reperfusion in mice. Lycopene preconditioning significantly improved the TMS and reduced neuronal death 

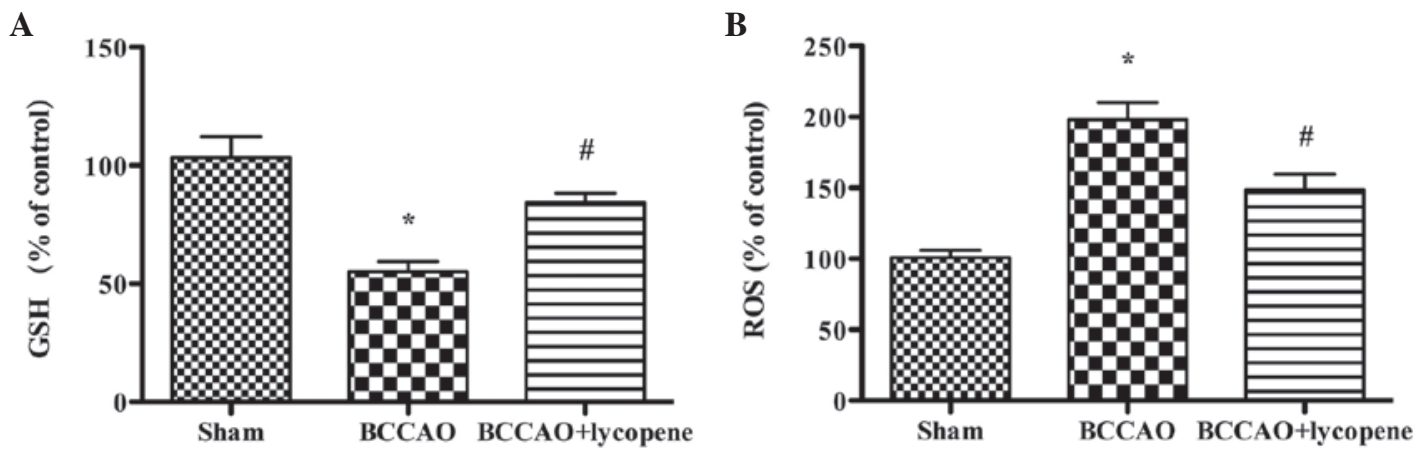

Figure 4. Production of (A) GSH and (B) ROS in each group. As shown, global cerebral ischemia led to a decrease in GSH and an increase in ROS. Lycopene treatment attenuated the production of ROS and enhanced the production of GSH ( $\mathrm{n}=10 ;{ }^{*} \mathrm{P}<0.05$ vs. Sham group; ${ }^{\text {}} \mathrm{P}<0.05$ vs. BCCAO group). GSH, glutathione; ROS, reactive oxygen species; BCCAO, bilateral common carotid artery occlusion.

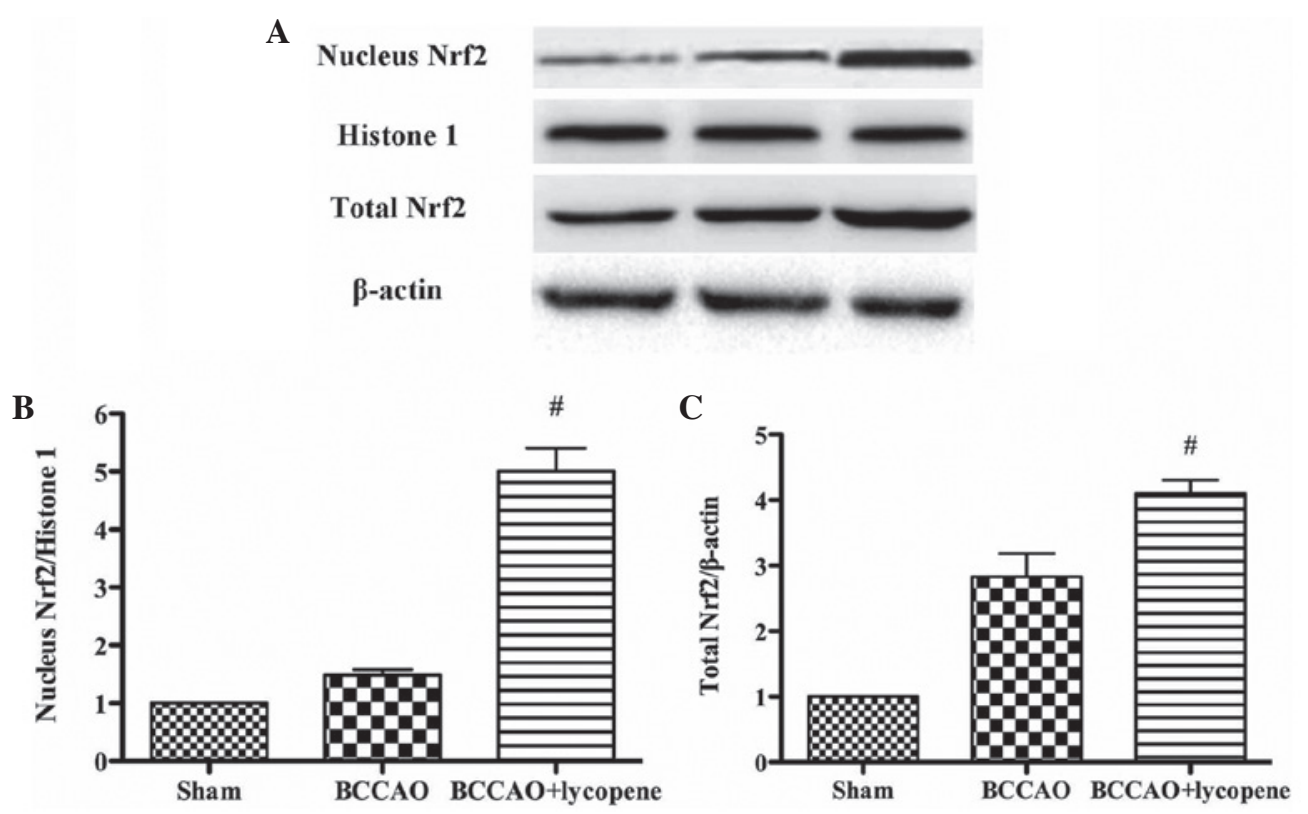

Figure 5. Lycopene treatment significantly upregulates the expression of nuclear and total Nrf2 in mice subjected to BCCAO. (A) A representative western blot, and the quantification of the data for (B) the nuclear Nrf2 measured against histone 1 and $(\mathrm{C})$ the total $\mathrm{Nrf} 2$ measured against $\beta$-actin ( $\mathrm{n}=10$; " $\mathrm{P}<0.05$ vs. Sham group, ${ }^{\sharp} \mathrm{P}<0.05$ vs. BCCAO group). BCCAO, bilateral common carotid artery occlusion; Nrf2, nuclear factor erythroid 2-related factor 2.

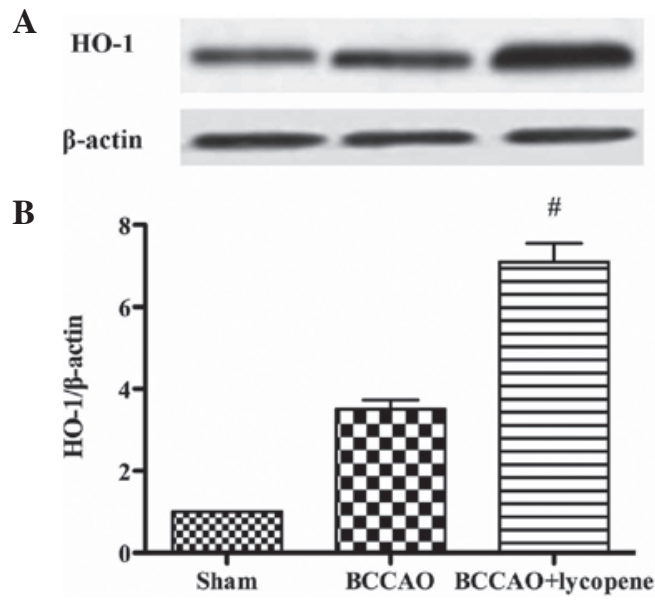

Figure 6. Lycopene treatment significantly upregulates the expression of cytoplasmic HO-1 in mice subjected to BCCAO. (A) A representative western blot and (B) the quantification of the data $\left(\mathrm{n}=10,{ }^{*} \mathrm{P}<0.05\right.$ vs. Sham group, ${ }^{\prime \prime} \mathrm{P}<0.05$ vs. BCCAO group). $\beta$-actin was used as an internal loading control. BCCAO, bilateral common carotid artery occlusion; HO-1, heme oxygenase-1. following cerebral ischemia-reperfusion. It was revealed that lycopene pretreatment induced an antiapoptotic effect and an antioxidative stress effect, which is demonstrated by its ability to increase the production of GSH and to decrease the production of ROS. In addition, lycopene activated the expression of Nrf2 and HO-1 in this global ischemic model.

Pyramidal neurons in the hippocampal CA1 region are particularly vulnerable to ischemia. This region undergoes delayed neuronal death, often reported as apoptosis in collaboration with DNA fragmentation (28). In the present study, a model of BCCAO was established and it was subsequently determined that lycopene pretreatment protected the brain from the ischemic injury, which is associated with its antiapoptotic effect and its antioxidative stress effect.

Neuronal apoptosis is an important pathological process of ischemic stroke (29,30). Rabuffetti et al (31) demonstrated that the inhibition of apoptosis reduces ischemic injury. Although two pathways of apoptosis, extrinsic and intrinsic, have been recognized (32), the final phase of apoptosis execution, which 
includes activation of executioner caspases (e.g. caspase 3), is shared by each of these pathways (33). Lycopene, a member of the carotenoid family, is found predominantly in tomatoes and other red colored fruits (8). It has been reported that lycopene protects against apoptosis in hypoxia/reoxygenation-induced H9C2 myocardioblast cells (34). In addition, He et al (35) reported that lycopene attenuates inflammation and apoptosis in postmyocardial infarction remodeling. The protective effect of lycopene in retinal ischemia/reperfusion injury has been demonstrated. It has been suggested that lycopene reduces the apoptosis of cells in the ganglion cell layer (36). The present study is in agreement with these studies and the results suggested that lycopene attenuated neuronal apoptosis in global ischemic brain.

Oxidative stress is also significant in ischemic brain injury (37). In ischemia/reperfusion injury, ROS is markedly produced and the endogenous antioxidant system cannot eliminate many of them. As a result, ROS leads to lipid peroxidation and DNA damage. It has been suggested that lycopene protects pancreatic acinar cells against severe acute pancreatitis (38). Additionally, lycopene prevents experimental priapism against oxidative damage, as reported previously (39), and attenuates oxidative stress in fructose-drinking insulin resistant rats (40). Consistent with these studies, the present study suggested that lycopene attenuates oxidative stress induced by global ischemia in the brain.

$\mathrm{HO}-1$ is a rate-limiting enzyme, catalyzing the degradation of heme into carbon monoxide, biliverdin and ferritin (41). HO-1 is regulated by the transcription factor Nrf2 at the transcriptional level (42). Under physiological conditions, Nrf2 is located in the cytosol by binding to Keap1 (43). In the presence of ROS, Nrf2 is released from Keap1 and translocates into the nucleus, activating the transcription of $\mathrm{HO}-1$. In the present study, brain ischemia/reperfusion injury leads a dramatic increase in the generation of ROS. Consequently, nuclear Nrf2 was increased and HO-1 was upregulated following ischemia-reperfusion injury. In addition, lycopene pretreatment significantly induced an increase in the expression levels of Nrf2 and HO-1.

In conclusion, these findings suggested that lycopene provided significant neuroprotection in mice subjected to global cerebral ischemia by inhibiting neuronal apoptosis and attenuating oxidative stress, which is associated with the activation of Nrf2/HO-1 signaling.

\section{References}

1. Li M, Qu YZ, Zhao ZW, Wu SX, Liu YY, Wei XY, Gao L and Gao GD: Astragaloside IV protects against focal cerebral ischemia/reperfusion injury correlating to suppression of neutrophils adhesion-related molecules. Neurochem Int 60: 458-465, 2012.

2. Amano M, Hasegawa M, Hasegawa $T$ and Nabeshima $T$ : Characteristics of transient cerebral ischemia-induced deficits on various learning and memory tasks in male Mongolian gerbils. Jpn J Pharmacol 63: 469-477, 1993.

3. Csiszar A: Anti-inflammatory effects of resveratrol: Possible role in prevention of age-related cardiovascular disease. Ann N Y Acad Sci 1215: 117-122, 2011.

4. Jung JE, Kim GS, Chen H, Maier CM, Narasimhan P, Song YS, Niizuma K, Katsu M, Okami N, Yoshioka H, et al: Reperfusion and neurovascular dysfunction in stroke: from basic mechanisms to potential strategies for neuroprotection. Mol Neurobiol 41: $172-179,2010$

5. del Zoppo GJ and Mabuchi T: Cerebral microvessel responses to focal ischemia. J Cereb Blood Flow Metab 23: 879-894, 2003.
6. Diaz-Ruiz A, Zavala C, Montes S, Ortiz-Plata A Salgado-Ceballos H, Orozco-Suarez S, Nava-Ruiz C, Pérez-Neri I, Perez-Severiano F and Ríos C: Antioxidant, antiinflammatory and antiapoptotic effects of dapsone in a model of brain ischemia/reperfusion in rats. J Neurosci Res 86: 3410-3419, 2008.

7. Armstead WM, Ganguly K, Kiessling JW, Riley J, Chen XH, Smith DH, Stein SC, Higazi AA, Cines DB, Bdeir K, et al: Signaling, delivery and age as emerging issues in the benefit/risk ratio outcome of tPA For treatment of CNS ischemic disorders. J Neurochem 113: 303-312, 2010.

8. Kuhad A, Sethi R and Chopra K: Lycopene attenuates diabetes-associated cognitive decline in rats. Life Sci 83: 128-134, 2008.

9. Sandhir R, Mehrotra A and Kamboj SS: Lycopene prevents 3-nitropropionic acid-induced mitochondrial oxidative stress and dysfunctions in nervous system. Neurochem Int 57: 579-587, 2010

10. Fujita K, Yoshimoto N, Kato T, Imada H, Matsumoto G, Inakuma T, Nagata $\mathrm{Y}$ and Miyachi E: Lycopene inhibits ischemia/reperfusion-induced neuronal apoptosis in gerbil hippocampal tissue. Neurochem Res 38: 461-469, 2013.

11. Ip BC, Hu KQ, Liu C, Smith DE, Obin MS, Ausman LM and Wang XD: Lycopene metabolite, apo-10'-lycopenoic acid, inhibits diethylnitrosamine-initiated, high fat diet-promoted hepatic inflammation and tumorigenesis in mice. Cancer Prev Res (Phila) 6: 1304-1316, 2013.

12. Palozza P, Simone R, Catalano A, Monego G, Barini A, Mele MC, Parrone N, Trombino S, Picci N and Ranelletti FO: Lycopene prevention of oxysterol-induced proinflammatory cytokine cascade in human macrophages: Inhibition of NF- $\kappa B$ nuclear binding and increase in PPAR $\gamma$ expression. J Nutr Biochem 22: 259-268, 2011

13. Qu M, Li L, Chen C, Li M, Pei L, Chu F, Yang J, Yu Z, Wang D and Zhou Z: Protective effects of lycopene against amyloid $\beta$-induced neurotoxicity in cultured rat cortical neurons. Neurosci Lett 505: 286-290, 2011

14. Yue R, Xia X, Jiang J, Yang D, Han Y, Chen X, Cai Y, Li L, Wang WE and Zeng C: Mitochondrial DNA oxidative damage contributes to cardiomyocyte ischemia/reperfusion-injury in rats: Cardioprotective role of lycopene. J Cell Physiol 230: 2128-2141, 2015.

15. Ip BC, Liu C, Ausman LM, von Lintig J and Wang XD: Lycopene attenuated hepatic tumorigenesis via differential mechanisms depending on carotenoid cleavage enzyme in mice. Cancer Prev Res (Phila) 7: 1219-1227, 2014.

16. Khachik F, Carvalho L, Bernstein PS, Muir GJ, Zhao DY and Katz NB: Chemistry, distribution, and metabolism of tomato carotenoids and their impact on human health. Exp Biol Med (Maywood) 227: 845-851, 2002.

17. Bun S, Ikejima C, Kida J, Yoshimura A, Lebowitz AJ, Kakuma T and Asada T: A combination of supplements may reduce the risk of Alzheimer's disease in elderly Japanese with normal cognition. J Alzheimers Dis 45: 15-25, 2015.

18. Prakash A and Kumar A: Implicating the role of lycopene in restoration of mitochondrial enzymes and BDNF levels in $\beta$-amyloid induced Alzheimers disease. Eur J Pharmacol 741: 104-111, 2014.

19. Chen H, Yoshioka H, Kim GS, Jung JE, Okami N, Sakata H, Maier CM, Narasimhan P, Goeders CE and Chan PH. Oxidative stress in ischemic brain damage: mechanisms of cell death and potential molecular targets for neuroprotection. Antioxid Redox Signal 14: 1505-1517, 2011.

20. He M, Siow RC, Sugden D, Gao L, Cheng X and Mann GE: Induction of HO-1 and redox signaling in endothelial cells by advanced glycation end products: A role for Nrf2 in vascular protection in diabetes. Nutr Metab Cardiovasc Dis 21: 277-285, 2011.

21. Itoh K, Chiba T, Takahashi S, Ishii T, Igarashi K, Katoh Y, Oyake T, Hayashi N, Satoh K, Hatayama I, et al: An Nrf2/small Maf heterodimer mediates the induction of phase II detoxifying enzyme genes through antioxidant response elements. Biochen Biophys Res Commun 236: 313-322, 1997.

22. Alfieri A, Srivastava S, Siow RC, Modo M, Fraser PA and Mann GE: Targeting the Nrf2-Keap1 antioxidant defence pathway for neurovascular protection in stroke. J Physiol 589: 4125-4136, 2011

23. Dinkova-Kostova AT, Holtzclaw WD, Cole RN, Itoh K, Wakabayashi N, Katoh Y, Yamamoto Mand Talalay P: Direct evidence that sulfhydryl groups of Keap1 are the sensors regulating induction of phase 2 enzymes that protect against carcinogens and oxidants. Proc Natl Acad Sci USA 99: 11908-11913, 2002. 
24. Zhang DD: Mechanistic studies of the Nrf2-Keap1 signaling pathway. Drug Metab Rev 38: 769-789, 2006.

25. Wakabayashi N, Dinkova-Kostova AT, Holtzclaw WD, Kang MI, Kobayashi A, Yamamoto M, Kensler TW and Talalay P: Protection against electrophile and oxidant stress by induction of the phase 2 response: fate of cysteines of the Keapl sensor modified by inducers. Proc Natl Acad Sci USA 101: 2040-2045, 2004.

26. Zhang HP, Sun YY, Chen XM, Yuan LB, Su BX, Ma R, Zhao RN, Dong HL and Xiong L: The neuroprotective effects of isoflurane preconditioning in a murine transient global cerebral ischemia-reperfusion model: The role of the Notch signaling pathway. Neuromolecular Med 16: 191-204, 2014.

27. Homi HM, Mixco JM, Sheng H, Grocott HP, Pearlstein RD and Warner DS: Severe hypotension is not essential for isoflurane neuroprotection against forebrain ischemia in mice. Anesthesiology 99: 1145-1151, 2003.

28. Miyamoto O, Tamae K, Kasai H, Hirakawa H, Hayashida Y, Konishi R and Itano T: Suppression of hyperemia and DNA oxidation by indomethacin in cerebral ischemia. Eur J Pharmacol 459: 179-186, 2003.

29. Brouns R and De Deyn PP: The complexity of neurobiological processes in acute ischemic stroke. Clin Neurol Neurosurg 111: 483-495, 2009

30. Doyle KP, Simon RP and Stenzel-Poore MP: Mechanisms of ischemic brain damage. Neuropharmacology 55: 310-318, 2008

31. Rabuffetti M, Sciorati C, Tarozzo G, Clementi E, Manfredi AA and Beltramo M: Inhibition of caspase-1-like activity by Ac-Tyr-Val-Ala-Asp-chloromethyl ketone induces long-lasting neuroprotection in cerebral ischemia through apoptosis reduction and decrease of proinflammatory cytokines. J Neurosci 20: 4398-4404, 2000.

32. Chen Mand Wang J: Initiator caspases in apoptosis signaling pathways. Apoptosis 7: 313-319, 2002

33. Thornberry NA and Lazebnik Y: Caspases: Enemies within. Science 281: 1312-1316, 1998.

34. Chen F, Sun ZW, Ye LF, Fu GS, Mou Y and Hu SJ: Lycopene protects against apoptosis in hypoxia/reoxygenation induced H9C2 myocardioblast cells through increased autophagy. Mol Med Rep 11: 1358-1365, 2015.
35. He Q, Zhou W, Xiong C, Tan G and Chen M: Lycopene attenuates inflammation and apoptosis in post-myocardial infarction remodeling by inhibiting the nuclear factor- $\kappa \mathrm{B}$ signaling pathway. Mol Med Rep 11: 374-378, 2015.

36. He M, Pan H, Chang RC, So KF, Brecha NC and Pu M: Activation of the Nrf2/HO-1 antioxidant pathway contributes to the protective effects of Lycium barbarum polysaccharides in the rodent retina after ischemia-reperfusion-induced damage. PloS One 9: e84800, 2014.

37. Liu Y, Zhang L and Liang J: Activation of the Nrf2 defense pathway contributes to neuroprotective effects of phloretin on oxidative stress injury after cerebral ischemia/reperfusion in rats. J Neurol Sci 351, 88-92, 2015.

38. Lv JC, Wang G, Pan SH, Bai XW and Sun B: Lycopene protects pancreatic acinar cells against severe acute pancreatitis by abating the oxidative stress through JNK pathway. Free Radic Res 49: 151-163, 2015.

39. Ciftci O, Oguz F, Beytur A, Polat F, Altıntas R and Oguzturk H: Lycopene prevents experimental priapism against oxidative and nitrosative damage. Eur Rev Med Pharmacol Sci 18: 3320-3325, 2014.

40. Yin Q, Ma Y, Hong Y, Hou X, Chen J, Shen C, Sun M, Shang Y, Dong S, Zeng Z, et al: Lycopene attenuates insulin signaling deficits, oxidative stress, neuroinflammation, and cognitive impairment in fructose-drinking insulin resistant rats. Neuropharmacology 86: 389-396, 2014.

41. Siow RC, Sato H and Mann GE: Heme oxygenase-carbon monoxide signalling pathway in atherosclerosis: Anti-atherogenic actions of bilirubin and carbon monoxide? Cardiovasc Res 41: 385-394, 1999.

42. Wei Y, Gong J, Yoshida T, Eberhart CG, Xu Z, Kombairaju P, Sporn MB, Handa JT and Duh EJ: Nrf2 has a protective role against neuronal and capillary degeneration in retinal ischemia-reperfusion injury. Free Radic Biol Med 51: 216-224, 2011

43. Cheng X, Siow RC and Mann GE: Impaired redox signaling and antioxidant gene expression in endothelial cells in diabetes: a role for mitochondria and the nuclear factor-E2-related factor 2-Kelch-like ECH-associated protein 1 defense pathway. Antioxid Redox Signal 14: 469-487, 2011 